\title{
L'ambivalence de convenience : commodité ou proximité ?
}

Dominique Claisse

\section{(2) OpenEdition}

1 Journals

Édition électronique

URL : http://journals.openedition.org/asp/3935

DOI : 10.4000/asp.3935

ISSN : 2108-6354

Éditeur

Groupe d'étude et de recherche en anglais de spécialité

\section{Édition imprimée}

Date de publication : 1 décembre 1995

Pagination : 311-324

ISSN : 1246-8185

\section{Référence électronique}

Dominique Claisse, «L'ambivalence de convenience : commodité ou proximité ? », ASp [En ligne], 7-10

1995, mis en ligne le 03 novembre 2013, consulté le 10 décembre 2020. URL : http:// journals.openedition.org/asp/3935; DOI : https://doi.org/10.4000/asp.3935

Ce document a été généré automatiquement le 10 décembre 2020.

Tous droits réservés 


\title{
L'ambivalence de convenience : commodité ou proximité?
}

\author{
Dominique Claisse
}

1 L'enseignement de l'anglais de spécialité peut difficilement se faire indépendamment de l'enseignement spécialisé que reçoit l'étudiant en Techniques de commercialisation (IUT). Les liens entre les différentes matières sont nombreux: langue spécialisée fortement teintée d'anglo-saxon, notions de base qui ont pour la plupart leur origine outreAtlantique, demandes des enseignants de matières spécialisées ou d'étudiants euxmêmes... Les études comparatives entre les différentes formules de vente sont un des moyens de répondre à ces diverses sollicitations. La perception de failles linguistiques peut aussi parfois mener, au travers de l'analyse de termes ou de notions particuliers, à une meilleure prise de conscience d'une forme d'altérité commerciale et/ou culturelle. Sur la base d'échanges de connaissances, d'une sorte d'interactivité donc, le dialogue peut alors s'instaurer entre l'apprenant qui se spécialise dans l'étude de la matière commerciale et l'enseignant de l'anglais des affaires. Value, service, discount ou convenience sont quelques exemples de ces termes porteurs de sens.

2 Dans le cadre des recherches en mercatique et commerce il arrive que l'étude de concepts particuliers ouvre au linguiste et enseignant d'anglais de spécialité des perspectives fructueuses : la proximité ${ }^{1}$ est une notion qui, en raison du renouveau qu'elle connaît et des développements qu'elle engendre en France aujourd'hui, fait l'objet d'analyses approfondies. La proximité est affaire de commerce, certes, mais aussi aujourd'hui de soins ou d'emplois par exemple. On peut alors se demander à quoi correspond en anglais cette notion de proximité, en termes linguistiques tout d'abord. Un premier mot vient à l'esprit, convenience ${ }^{2}$. L'objectif de cette analyse n'est pas de dire que convenience et proximité ne sont pas équivalents : c'est une évidence. On peut toutefois penser que le concept de proximité trouve sa traduction commerciale la plus fidèle dans cette notion américaine, riche de connotations culturelles. Il ne s'agit donc pas de créer une opposition facile, factice, entre deux termes proches. Il convient de préciser aussi dès à 
présent que l'approche faite ici de convenience est fondée sur la dimension commerciale du mot.

\section{Convenience in situ}

3 Comment aborder ce terme? Une simple anecdote, banale et a priori insignifiante, permettra peut-être d'éclairer la démarche choisie: Washington, D.C., n'est pas, à première vue, l'endroit idéal où l'on peut tenter d'appréhender la réalité tangible de convenience. Les commerces en effet y sont à l'image de la ville, fonctionnels. Ils ne sont toutefois pas les géants annoncés qu'il faudra voir ailleurs. Ici pas de gigantesques malls, tels le Mall of America ou le Caesar's Palace ; il faut même sortir du cœur de la ville pour voir quelques centres commerciaux au luxe un peu tapageur. Le hasard toutefois fait parfois bien les choses. Il peut arriver au promeneur de se perdre dans le dédale des chemins. On se dit alors que la vie, celle que l'on dit courante ou de tous les jours, n'est pas si simple dans cet immense labyrinthe, et que l'homme de la rue doit éprouver bien des difficultés à satisfaire des besoins d'achats premiers, ceux qui rendent possible, agrémentent ou facilitent la vie. Des boutiques existent, pourtant, et il arrive que l'on tombe, tout à coup, entre deux immenses blocs de béton, sur l'une d'elles : c'est le cas de Stanby, située dans l'interminable $\mathrm{K}$ street au $\mathrm{n}^{\circ} 1201$. Elle a pour devise Lil'bit of Everything. Qu'y trouve-t-on? Littéralement « un peu de tout ». Le prospectus qu'on offre au passant intrigué est orné d'un dessin, celui d'un chien de prairie, un des nombreux animaux des plaines américaines, symbole de vie en communauté et de vigilance. On peut y lire : "Come and see us for your health, and beauty aids, snacks, souvenirs, T-shirts, postcards, maps, cigarettes, fresh fruits, newspapers and just about a Lil'bit of everything!». Ses heures d'ouverture sont de 8 heures du matin à 9 heures du soir sans interruption en semaine, de 8 heures à minuit les vendredi et samedi, et même le dimanche de 9 heures à 16 heures. Le service est assuré par une jeune fille de couleur qui disparaît à demi, comme avalée par un comptoir qui occupe à lui seul près du quart de la surface commerciale disponible.

Il s'agit bien d'une de ces boutiques connues sous le nom de convenience stores, ou magasin de proximité, à l'américaine. Voilà donc l'exemple, singulier à vrai dire, de cette notion commerciale, convenience, qui revient sans cesse au hasard des lectures de la presse spécialisée américaine, et, à un degré moindre, britannique. L'anecdote prend alors tout son sens : la convenance ${ }^{3}$ est peut-être ce à quoi on ne s'attend pas, la surprise agréable, proche et pratique à la fois ; ni contrainte ni construction complexe ou distante, elle est à portée de main, disponible, et il suffit d'un peu de chance pour la trouver. Ténue comme un fil d'Ariane, elle va être le guide qui conduira aux mots-clés, un peu comme une idée qui va au gré des mots et les relie parfois. Elle mérite qu'on s'y attarde.

Cette analyse prendra la forme d'un cheminement qui mènera d'une exploration linguistique à une étude commerciale du concept lui-même. On peut dès à présent parler non pas d'étapes distinctes mais bien davantage d'une sorte de continuum entre occurrences de nature linguistique, considérations commerciales que traduisent les mots abordés et phénomènes culturels sous-jacents. 


\section{Un mot qui se dérobe}

6 Le terme convenience est difficile à cerner pour diverses raisons : un effeuillage s'impose. C'est tout d'abord un mot qui a connu au cours des années passées de nombreux glissements sémantiques. Il y a deux décennies on le trouvait sous la forme convenience goods. Il s'agit alors des produits de consommation courante disponibles dans le magasin de proximité. Cette dénomination est déjà le signe d'une volonté de rationalisation et nécessite une connaissance approfondie des attitudes des consommateurs. On les distingue alors des shopping goods (produits à achat réfléchi) et des specialty goods (produits spécialisés) ${ }^{4}$. Ces convenience goods sont l'apanage des convenience stores, connus aujourd'hui souvent par leur abréviation - comble d'insignifiance pour le mot qui fait l'objet de cette étude - les c-stores ${ }^{5}$. On peut noter ici que les convenience stores peuvent être aussi bien des magasins traditionnels, représentant ce que l'on appelait naguère le petit commerce, que les innombrables drugstores américains ou autres surfaces de vente de proximité. Un premier glissement apparaît en français: les convenience goods deviennent produits banals et s'opposent aux produits anomals (anomalous products); banals semble parfois gêner certains utilisateurs du mot par sa connotation péjorative (absente dans convenience goods) et il n'est pas rare d'entendre ou de lire l'autre pluriel de banal, banaux, qui permet de remonter à la racine du mot. Les convenience goods ${ }^{6}$ sont des produits de grande consommation et ce qui les distingue alors c'est leur disponibilité ou leur accessibilité. Leur prix doit être le plus bas possible pour servir parfois de produits d'appel (loss leaders). Ils rapportent peu au commerçant : leur domaine n'est pas la marge, mais le volume ${ }^{7}$. Ils se limitent aux produits de première nécessité et s'apparentent aux produits génériques $^{8}$. Ils forment les produits de base (staple goods), qui se distinguent des produits d'intérêt secondaire (ancillary products).

7 Le mot s'échappe ; non content de se faire reconnaître d'une seule lettre, il s'associe au gré du temps et des modes. On trouve convenience aujourd'hui en compagnie de substantifs variés. Le c-store était d'abord lié à l'idée de proximité : nearness, closeness ou encore neighborhood par exemple, en compagnie desquels on le trouve souvent, de même que proximity, plus rare, mais que l'on rencontre parfois ${ }^{9}$. On peut dès à présent supposer que la traduction française couramment acceptée de convenience (dans convenience store) par proximité représente un cadre trop rigide : la dimension spatio-temporelle du mot français - la distance entre le consommateur et le point de vente et le temps qu'il faut pour la parcourir - ne recouvre, semble-t-il, qu'une partie du terme ${ }^{10}$. Le magasin de commodité ou de convenance est à l'origine le magasin du coin de la rue (corner shop), très facile d'accès et qui dispose d'un assortiment (assortment) large et peu profond. Il est magasin de dépannage (top-up shop) et permet des achats du même nom (top-up shopping). Il se distingue alors du centre commercial ou du mall qui deviennent, eux, des destination stops (magasins d'attraction), ou endroits où l'on va pour effectuer ses achats principaux ( primary shopping).

8 Convenience, c'est donc bien ce Lil'bit of everything, que l'on trouve dans le magasin Standby, au $1201 \mathrm{~K}$ Street, à Washington. On croirait le mot logé, encadré pour ainsi dire.

9 Que penser alors de l'intensif qu'on lui accole parfois, le super-convenience store, aux horaires plus larges et au plus grand choix de produits? On peut aussi considérer que le neighborhood shopping center s'en approche, de même que le plus explicite encore convenience center. 
10 Il y a déplacement dans l'espace, donc, après le déplacement dans le temps. L'évolution des divers sens du mot au cours des deux dernières décennies est le signe du changement, de la progression d'un terme à la sémantique déroutante. Peut-être peut-on désormais rechercher les mots qui l'entourent, son cercle de famille, ceux auxquels on l'associe et dans l'entourage desquels il accepter de rester, pour un certain temps du moins. On verra en tout cas que l'acception classique de convenience goods est en partie dépassée.

\section{Le mot et ses alliances}

11 On a déjà évoqué dans la deuxième partie les rapprochements avec des termes comme consumer (dans consumer goods), shopping, shopping goods et d'autres. Plus révélatrice peutêtre est la proximité du terme ease dans l'entourage de convenience ${ }^{11}$. Ce mot parle de flexibilité, de simplicité, de naturel et d'insouciance. Les convenience products ou comfort products (produits de confort) évoquent eux, outre la facilité, à nouveau l'opulence, la richesse ou le bien-être. On va plus loin encore quand on analyse l'emploi le plus courant aujourd'hui de convenience, dans convenience foods (produits alimentaires de confort; plats préparés) ou convenience drinks (boissons prêtes à consommer). Ces produits alimentaires représentent l'ensemble des plats préparés, prêts à la consommation et qui répondent à des exigences de vitesse, de commodité et de qualité. Le prix n'est plus leur attrait essentiel.

On évoquera en revanche leur rapport qualité-prix (value-for-money, value). Il peut s'agir de légumes ou fruits pré-coupés (pre-cuts) et pré-emballés (pre-packaged goods), de boissons qui offrent des mélanges tout prêts, ou encore et surtout des produits que l'on trouve dans le rayon traiteur, avec ou sans service (self-service deli, service deli).

13 Confort, rapidité et souplesse d'utilisation, flexibilité donc ; mais le terme évoque aussi la qualité, la fraîcheur, une décision d'achat que l'on accepte d'être plus longue, et un prix que l'on sait être plus élevé mais que l'on admet car vendu avec service rendu. Le passage de la notion de banalité à celle de service est une autre de ces clés qui permettent de mieux cerner le mot analysé. Les convenience products sont, aux États-Unis au moins, l'apanage des supermarchés bien davantage que des c-stores ou autres drugstores ; le mot permet cette fois de changer de magasin. Ce passage d'une commodité fondée sur l'emplacement à une commodité fondée sur le choix d'une formule commerciale apparait en anglais, qui distingue aujourd'hui convenience of location et convenience of store.

14 Ce terme est décidément bien élusif et la réponse recherchée n'est donc que partielle quand on l'associe à d'autres. Insaisissable, il l'est dans l'espace et dans le temps, dans son environnement aussi. Certains spécialistes en mercatique parlent de convenience comme d'un avantage intangible :

Intangible Service Benefits: aspects such as comfort, efficiency, convenience and rapidity are seldom codified as categories by the consumer, but are rather viewed by each individual according to his own parameters, usually on the basis of comparison with past experiences. (Cristini $1992: 198$ )

15 Il s'agit ici d'une tentative de définition de nature commerciale, qui permet de dépasser la simple approche linguistique. 


\section{Convenience ou le signe de la polarisation}

16 La polarisation ${ }^{12}$ est dans l'esprit de nombreux spécialistes la tendance majeure des années à venir. Le mouvement est double et tend vers les extrêmes. D'un côté des surfaces de vente spécialisées géantes qui attirent les clients avec des prix toujours plus bas. C'est aux États-Unis l'invasion remarquée depuis quelques années des hard discounters (bébésrequins, casseurs de prix), category killers (tueurs de catégorie) et autres warehouse clubs (clubs-entrepôts), regroupés parfois en power centers (centres d'énergie). Les clients vont vers eux en masse, effectuer là l'essentiel de leurs achats et profiter de prix qui leur permettent de mieux résister à la crise. Ces prix ne sont plus de simples promotions mais suivent désormais une politique constante de prix bas (Everyday Low Prices; EDLP ou ELP). Les points de vente sont hors des villes, véritables nœuds commerciaux à la jonction des principales voies d'accès, et desservent une très vaste zone de chalandise (catchment area). Le client y trouve les moyens de sa mobilité (essence), de sa restauration et de son approvisionnement en marchandises diverses; c'est aussi le lieu où d'exprime l'instinct grégaire par excellence, celui où l'on s'assemble, qui permet un retour au sens étymologique de convenience (1).

17 Considérations financières donc, gigantisme aussi, tant dans les surfaces construites que dans la taille des emballages (jumper pack, bumper pack); risque de déshumanisation enfin.

Ces grandes surfaces de vente revendiquent aussi la notion de convenience; on parlera souvent à leur endroit de "one-stop-shopping convenience » (commodité des achats en un seul lieu), à l'image de cette cliente américaine à qui on demandait son avis sur la taille d'un gigantesque hypermarché à l'américaine et qui s'exclama That's convenience! Le client y est certes encore le roi, mais un roi anonyme, élément d'une foule qui seule justifie l'existence de ces centres.

19 Le mouvement évoqué est celui des clients vers les grands centres commerciaux. Il existe, un peu comme en retour, un mouvement inverse, véritable courant lui aussi, moins spectaculaire certes mais tout aussi révélateur. C'est celui du développement ou du renouveau du commerce de proximité, du petit supermarché spécialisé dans les produits frais, du magasin commode, aux heures d'ouverture qui permettent un accès facile à tous. C'est le lieu convivial où le prix importe moins que le service, qui devient, lui, primordial ; le lieu de vente vient au client, en quelque sorte. Le magasin de commodité a changé et il s'est adapté. On y trouve même souvent les améliorations modernes de la technique : le code à barres (bar-code) et son lecteur optique (scanner), le paiement par carte (Electronic Point of Sale, EPoS), par exemple. Cette forme de magasin s'est transformée et revit, elle aussi, au travers de la notion de convenance.

20 À l'origine qualité essentielle du magasin qui porte son nom, convenience s'est échappé de ce cadre trop rigide et, sollicité par toutes les formes de commerce, il prend tout son sens quand on l'associe au déplacement qu'effectue chacun pour relier son lieu de vie à son lieu de travail :

Convenience now means anything from «near to work», «on the way home",

« close to a large shopping centre », to « near to home ». (Retail Week $1993: 19$ )

21 Convenience n'est plus restreint à un type de produits ou à une formule commerciale. S'il y a exclusivité, c'est désormais bien celle du service au citoyen-consommateur. Il faut bien admettre alors que l'approche commerciale, si elle permet d'entrer plus avant dans les 
arcanes d'un mot aussi intangible et d'en voir le double mouvement, requiert de passer à l'étape suivante, de nature culturelle cette fois

\section{La philosophie de la convenance}

L'expression existe bien et se trouve sous cette forme, convenience philosophy. Au cœur de cette approche, de cette philosophie donc, on trouve bien sûr le consommateur, qui en est l'agent essentiel. Tout est fait pour lui plaire, pour lui simplifier la vie et l'on parlera alors de produits qui sont consumer-friendly (convivial), user-friendly, people-friendly ou encore shopper-friendly. Les détaillants sont désormais tenus d'offrir non seulement le confort ou l'aisance, mais aussi de montrer la connaissance intime qu'ils ont du consommateur ( customer intimacy) ${ }^{13}$.

Parler d'intimité permet ici d'introduire la notion de téléachat, qui procède d'une démarche où interviennent également les notions de commodité et de proximité. L'activité de magasinage que l'on effectue de chez soi par le biais de moyens de communication nouveaux évoque plutôt le terme cocooning, ou sa version récente le burrowing.

Le coconnage, s'il est bien sûr, lui aussi, la conséquence d'une exigence de commodité, de confort ou de convenance, diffère du magasin de commodité en ce qu'il suggère aussi une forme de repli sur soi ou d'isolement. Le retour au terrier est, lui, franchement le signe d'une fermeture, ou pire, d'un enfermement. Par certains côtés, il pourrait être le signe de la rétraction face à la dangerosité d'une société. La convenance, elle, reste conviviale. Elle est inhérente à la notion de plaisir, le plaisir d'acheter, de voir et de toucher ${ }^{14}$ de vivre pleinement la relation commerciale à l'autre et elle transforme le magasinage en agrément ${ }^{15}$. À la tâche banale, qui s'apparente parfois à une corvée (chore), se substitue désormais, au gré du client, l'assouvissement d'un besoin qui, partant, devient source de satisfaction, pour peu qu'on laisse le client libre de choisir, d'aller et de venir, qu'on le laisse faire comme il l'entend. On peut dès lors évoquer les contraires de convenience pour aider à définir la notion : la contrainte bien sûr, mais aussi le verbe importuner ${ }^{16}$.

Par son côté urbain, la convenance se veut réponse à des besoins divers ; elle est parfois aussi signe d'une spécificité. Il n'est pas rare de voir les c-stores tenus par des minorités qui voient là un moyen de s'intégrer à la société d'accueil. C'est vrai aux États-Unis, mais aussi en Europe, où nombre de petits magasins de ce type sont gérés par des personnes venues des dernières vagues d'immigration. Elle est singulière, parfois signe d'exception, différence, à l'image non plus cette fois de la foule mais des personnes qui la composent. Ses clients sont souvent ceux qui n'entrent plus (les retraités) ou pas tout à fait (les mères célibataires qui travaillent par exemple) dans un moule normatif. Elle respecte davantage, se déplace vers les gens. Elle est complémentaire, indispensable donc. Peut-être est-elle même un peu un correctif ou un élément sécurisant dans une société en crise. Elle permet en tout cas une sorte de retour à l'expression de la liberté individuelle.

La convenance est avant tout le signe d'une modernité. Comment mieux la représenter que par le congélateur, et surtout le four à micro-ondes, cette machine moderne qui associe vitesse, gain de temps, mode de vie nouveau, économie d'usage et liberté de la ménagère? Cet appareil voit lui aussi des termes neufs se créer quand on l'évoque: microwave-ready (prêt à cuire en four à micro-ondes), microwaveable (micro-ondable, utilisable en four à micro-ondes), microwaveability (micro-ondabilité, aptitude à être 
utilisé en four à micro-ondes), microwave-specific ou micro-specific (spécial micro-ondes). Comment mieux qualifier cette invention qu'avec ce néologisme savoureux, les homey products (produits " faits comme à la maison »), idéal culinaire des time-starved shoppers (clients pressés, en manque de temps) ? Le four à micro-ondes semble être à bien des égards l'outil, ou la clé, qui a permis le développement du terme convenience.

\section{Influence américaine}

Le c-store est américain, le four à micro-ondes aussi. Force est de constater que le terme convenience foods est né aux États-Unis, comme avant lui le terme convenience goods. La convenance est certes l'un des états d'esprit du Pragmatisme, ou philosophie à l'américaine. Les mots-clés n'ont pas manqué : argent, prix, facilité, rapidité, efficacité, progression, évolution, service, renouveau et tant d'autres encore. L'influence semble claire et il est bien difficile de l'ignorer. L'Amérique est aussi le pays des exemples concrets qui sont analysés et dont il devient possible de déduire des règles plus globales. C'est un peu le sens du récit du début de cette étude. Mais au-delà de l'anecdote riche de sens, on peut en guise de conclusion mettre en lumière deux faits qui résument les points essentiels qui viennent d'être étudiés et ouvrent de nouvelles perspectives de réflexion.

Le magazine LSA (1992 : 41) consacrait récemment une série d'articles au renouveau du commerce de proximité. Après avoir fait remarquer que l'Europe, donc la France, ne dispose plus que de 65 magasins de détail pour 10000 habitants, - alors que les ÉtatsUnis en avaient encore, eux, 75 - cette étude se livre à une analyse approfondie des mouvements en cours en France. Parmi ceux-ci, certains présentent des similitudes avec l'évolution des convenience stores américains : restructuration indispensable, mise au point d'une politique commerciale du commerce de proximité et ses corollaires, le repositionnement continu et l'adaptation permanente aux besoins des clients, mais aussi changements dans la composition de la clientèle (personnes seules, femmes qui ont un emploi, accroissement du nombre de personnes âgées), importance du nombre de commerces de proximité tenus par des membres des minorités (maghrébine surtout). Tous ces points sont communs aux deux continents. Deux articles concluent cette étude et montrent une fois encore l'influence anglo-saxonne. L'un, intitulé « le convenience store a tout'heure ", parle d'une redéfinition du concept de proximité, adaptation d'une idée anglaise. On y évoque les îlots d'encaissement et les nombreux services: 82 heures d'ouverture hebdomadaires, mais aussi location de cassettes vidéo, vente de boissons fraîches à l'unité, un point fax, la vente de cartes de téléphone, livraison à domicile.

Peut-on imaginer meilleure description de la proximité au service des gens, de la proximité faite commodité en un mot? Significatif encore le titre du second de ces articles qui affiche "Les convenience stations, l'exemple américain" (LSA 1992: 50). La confusion semble bien régner entre commodité et proximité et les distinctions qui y sont faites laissent perplexe : «Nous parlons de magasins de commodité pour les petits et de proximité pour les plus grands », affirme J.-P. Adam, responsable des magasins de station essence Esso. Et le journaliste de poursuivre :

On [en] distingue deux sortes. Les magasins de commodité, des supérettes de 45 à $80 \mathrm{~m} 2$, et les convenience stores qui, sur des surfaces supérieures à $100 \mathrm{~m} 2$, proposent des gammes alimentaires, des produits frais et des services. 

on peut lire : convenance.

C'est donc le renouveau en France comme aux États-Unis d'une forme de commerce aux spécificités fortes et à l'avenir prometteur. On ne peut manquer de noter ici la perplexité, voire la confusion, dans l'emploi des termes.

On peut remarquer ici que les Canadiens français, à l'imagination si fertile en matière linguistique, proposent le mot dépanneur pour traduire convenience store. Ce terme est même recommandé par l'Office de la langue française du Québec (Gazette Officielle du Québec, 19 Mars 1993).

Le sentiment de malaise linguistique, de culture commerciale différente et d'influence qui s'exprime par le biais des mots, est également apparu dans un article récent publié dans Le Monde, sous le titre "Le réveil ambigu du luxe » (5 avril 1994). Au chapitre "Confort »,

Croissance lente. Consommateur exigeant. Pression sur les prix. Nouvelle forme du luxe, moins proche peut-être du raffinement cher à la tradition française que de cette recherche, très anglo-saxonne, d'un certain confort (le « convenient »).

Le glissement du raffinement à la française au confort à l'américaine est fécond en matière de réflexion culturelle, bien davantage que le recours au terme américain pour exprimer la notion de convenance.

Un autre point ouvre des perspectives nouvelles et relie l'objet de cette étude, convenience, au thème majeur du commerce moderne, la valeur (value). La convenance est un service essentiel que le consommateur achète ; elle prend diverses formes qui ont été analysées plus haut. Tous les types de commerce veulent se l'approprier, comme s'il s'agissait d'un mot magique, un nouveau sésame commercial en quelque sorte. Loin d'être un élément rapporté, elle fait partie des exigences du consommateur moderne. À l'avenir, il est possible d'ailleurs que soit refusée l'alternance entre prix et services, dont fait partie la

Selon certains professionnels, le commerce à venir sera peut-être la meilleure combinaison possible entre les trois exigences du consommateur, à savoir la qualité, le prix et le service, et quelle que soit la formule commerciale choisie ou la politique commerciale poursuivie, ces trois exigences devront être respectées pour la meilleure satisfaction du consommateur. Dans cette optique, convenience devient une valeur sûre et permet d'aller au-delà du mouvement actuel vers une polarisation du marché : le choix service ou prix est ainsi dépassé, le client exigeant les deux sans avoir à sacrifier l'un ou l'autre. Comme on pouvait le lire récemment dans Stores (1993, RR4) :

Value has come to mean much more than just price; it now also includes product relevance, fashionability, quality, service and convenience.

Le client américain, demain le client européen, dépasse la préoccupation du prix (priceconscious consumer) et devient plus exigeant; son attitude donne naissance à des termes qui apparaissent de plus en plus souvent comme value-conscious customer ou value-oriented consumer. Enfin, peut-être peut-on citer une phrase devenue célèbre de Sam Walton, le créateur de Wal-Mart, première chaîne commerciale au monde, qui procède par la négative dans son approche de la convenance, et s'adresse au client en ces termes :

You love it when you visit a store that somehow exceeds your expectations, and you hate it when a store inconveniences you, or gives you a hard time, or just pretends you're invisible. (Retail Week, $1992:$ I, 23, V, 5,5)

Si convenience est désormais une valeur, elle l'est aux yeux du consommateur qui seul peut décider quel produit ou quel magasin la mérite. On pourrait presque parler de label de 
qualité, octroyé selon des critères de convenance de l'acheteur, de convenance personnelle donc.

\section{Conclusion}

39 Le débat interculturel est sans fin qui tend à comparer deux mots, convenience et proximité, lesquels recouvrent des perspectives et un sens parfois bien éloignés.

Cette étude a tenté de montrer que l'usage d'un mot était rarement innocent ou gratuit. Une des difficultés principales dans l'appréhension du terme convenience vient du fait qu'il est en constante évolution. Les étapes de sa progression ont été notées au cours de l'analyse. Il est tout d'abord dépendant des mots store et goods. Associé à goods il est synonyme d'ordinaire et d'anonyme: les convenience goods sont des produits souvent génériques, connus non pas par une marque mais par une composition, pâtes, riz ou farine par exemple. Manque de distinction, donc, pris dans son sens étymologique !

41 Le recours en français au sens premier de banal (qui bénéficie du droit de banalité), dont le pluriel est cette fois banaux, est peut-être le signe d'une forme d'appréciation, pour employer un anglicisme riche de sens. On tente par là de gommer toute connotation péjorative. Simple initiale dans c-store, le mot passe pour ainsi dire inaperçu ; au gré des associations de termes ou d'idées on le retrouve toutefois bientôt sous d'autres formes où il suggère alors une forme de service : synonyme de confort, il se trouve dans un nouveau contexte, redécouvert, puis revendiqué par tous. Il devient concept. Convenience est signe de rassemblement d'une part, de l'expression d'une préférence individuelle d'autre part: peut-être est-il même symbole de l'individualisme "à l'américaine » ? Sa progression est semble-t-il parallèle à l'importance croissante qu'a au cours des années acquise le consommateur américain.

42 Cette analyse a essayé également de mettre en relief la difficulté qu'il y a de trouver en français un équivalent exact à convenience. Le terme proximité convient dans certains cas, pas dans d'autres. Il n'est pas plus pauvre que convenience et le recours à une acception classique du terme - celle de parenté - pourrait lui donner une dimension nouvelle. La proximité devient alors l'expression du rassemblement en un même lieu de gens proches par leurs besoins ou leurs désirs, signe d'un sentiment qui est peu apparu au cours de cette étude, celui du groupe, de la communauté, de l'existence et de l'importance de l'autre ${ }^{17}$, alors que convenience exalte surtout le consommateur en tant qu'individu: rechercher et trouver "ce qui me convient » est source de satisfaction et de bien-être individuel. La convenance n'en est peut-être qu'à une étape de son développement linguistique et culturel, et peut s'enrichir encore, de ce sens classique de proximité, par exemple.

Le magasin de proximité redevient alors magasin des proches, qu'ils le soient dans l'espace qui les sépare les uns des autres ou en raison du temps qu'il faut pour qu'ils se retrouvent; mieux encore, il permet de redevenir lieu d'échange, de rencontres ou de " communication », terme employé pour sacrifier à la mode linguistique. Par ce retour, ou recours, à l'acception classique d'un mot, on en revient alors aux origines du mot commerce : "achat et vente de marchandises » ou "lieu où s'effectue la transaction ", certes, mais aussi : "ensemble des relations sociales entre les personnes", ou encore "système de relations réciproques ", échange donc: le phénomène est commun à toutes les époques, à tous les lieux. En tant qu'expression d'une culture dominante, convenience 
est temporaire : c'est la version américaine d'un fait commercial de la fin du vingtième siècle.

\section{BIBLIOGRAPHIE}

Collins. 1987. The Collins Dictionary and Thesaurus. Londres : Collins.

Cristini, G. 1992. « Service quality in non-grocery distribution ». The International Review of Retail, Distribution and Consumer Research 2/2.

Dictionnaire de la Langue Française. 1989. Paris : Larousse.

Hall, E. 1971. La Dimension cachée. Paris : Seuil, Points.

LSA 1031. 23 avril 1992.

Retail Week, 24 July. 1992.

Retail Week, 26 Nov. 1993.

Stores, October 1993.

The Oxford Library of Words and Phrases. III Word Origins. 1986. Londres : Guild Publishing.

\section{NOTES}

1. Proximité : (du latin proximitas, de proximus : très proche) situation d'une chose qui est à peu de distance d'une autre, qui est rapprochée dans le temps. Syn : voisinage, contr. : éloignement ; classique : parenté (Larousse 1989 : 1525).

2. Convenience: the quality of being suitable or opportune. (Collins Thesaurus 1987: 213): accessibility, appropriateness, availability, serviceability, usefulness (ibid.). Convene (to) : come (to) together. Latin : convenire : assemble (to). (Oxford Words and Phrases 1986 : 96).

3. Convenance : qualité de ce qui convient à quelqu'un ou à quelque chose. Syn. : gré ; termes proches : fantaisie, affinité ; commodité, facilité, confort; en langage commercial : proximité, commodité (Larousse Dictionnaire 1989 : 422.

4. Ces trois catégories sont parfois nommées commodity groups.

5. Le terme possède également un négatif : non-convenience store.

6. On parlera à leur sujet de ordinariness of goods.

7. Produit à fort écoulement : traffic draw, volume builder ; produit qui génère des bénéfices : profit generator.

8. generic products ou generics; syn : no-frill goods; economy goods.

9. On doit évoquer à ce stade de l'étude les travaux menés aux États-Unis par E. Hall, relatifs à la 'proxémie'. «Le terme de proxémie est un néologisme que j'ai créé pour désigner l'ensemble des observations et théories concernant l'usage que l'homme fait de l'espace en tant que produit culturel spécifique » (1971: 13).

10. On doit remarquer ici qu'en anglais, et pour des raisons peut-être similaires, le terme d'origine latine proximity (de même que son proche voisin vicinity) ne porte pas de charge 
affective suffisante pour être couramment employé. On lui préférera les termes nearness ou closeness.

11. Ease of access : facilité d'accès ; ease of picking : facilité de prélèvement ; ease of shopping : facilité de magasinage.

12. Market polarisation : il s'agit du mouvement qui accentue le phénomène de polarité (polarity of retail trade).

13. Les travaux de T. Hall ont montré également dans ce domaine des différences d'approche conséquentes entre les cultures occidentales. Les phénomènes d'intrusion ou de sphère privée $\mathrm{y}$ sont clairement analysés. Dans sa théorie de la structuration de l'espace, il distingue par exemple l'approche française (et espagnole), qualifiée d'étoile ou système "sociopète " (en ce qu'il relie entre eux tous les points et toutes les fonctions), et l'approche britannique, en forme d'échiquier ou «sociofuge ». Cette interprétation de la centralisation "à la française » ne manque pas de saveur et illustre bien le fait que les différences existent non seulement dans la façon de dire les choses mais aussi de les ressentir, et donc de les faire.

14. "The touchy, feely aspect of retailing ".

15. "The leisure experience ».

16. Hassle-free : l'expression qualifie un acte d'achat sans sollicitation excessive et importune de la part du vendeur.

17. On retrouve cette dimension humaine dans l'emploi des termes « soins de proximité, emplois de proximité ».

\section{RÉSUMÉS}

Pris dans son sens commercial, convenience permet de relier des considérations aussi diverses que l'évolution linguistique d'un mot, les faits commerciaux qu'il souligne et les implications culturelles qu'il révèle. À l'origine synonyme de proximité, voire de banalité, le terme fait aujourd'hui référence à une forme de service, pour devenir enfin une des composantes de value. Il est aussi le signe d'un mode de vie, "à l'américaine ", symbole de liberté individuelle. Il traduit une autre culture de consommation qui fait peu à peu sentir ses effets sur le commerce français.

In its commercial sense, convenience may be a means to relate such considerations as the linguistic evolution of a word, the commercial facts it highlights and the, underlying cultural phenomena it reveals. Originally an element of proximity and a synonym for common, the word also refers now to a form of service, and has eventually become a component of value. It is also a token of the American way of life and a symbol of individual freedom. It conveys another consumer culture, which gradually bears upon French retailing.

\section{INDEX}

Keywords : consumption, convenience, retailing

Mots-clés : commerce de détail, convenance, consommation, culture 


\section{AUTEUR}

\section{DOMINIQUE CLAISSE}

Dominique Claisse enseigne à l'IUT de l'Université de Valenciennes et du Hainaut-Cambrésis, dans le département Techniques de commercialisation. Dominique.Claisse@univ-valenciennes.fr 